\title{
On a spectral theorem for deformation quantization
}

\author{
B. Fedosov
}

\begin{abstract}
We give a construction of an eigenstate for a non-critical level of the Hamiltonian function, and investigate the contribution of Morse critical points to the spectral decomposition. We compare the rigorous result with the series obtained by a perturbation theory. As an example the relation to the spectral asymptotics is discussed.
\end{abstract}

AMS subject classification 2000: 53D 55

Key words: star-product, WKB method, spectral theorem.

\section{Introduction}

Let us first remind the spectral theorem for a self-adjoint operator $H$ on a Hilbert space. It reads that there exists a spectral family $E_{\lambda}$ of increasing projectors commuting with $H$ which gives a partition of unity

$$
\int_{-\infty}^{\infty} d E_{\lambda}=1
$$

and a spectral decomposition of $H$ :

$$
\int_{-\infty}^{\infty} \lambda d E_{\lambda}=H .
$$

Applying both sides of the first equality to an arbitrary trace class operator $A$ and taking traces, we obtain

$$
\int_{-\infty}^{\infty} d\left(\operatorname{Tr} E_{\lambda} A\right)=\operatorname{Tr} A .
$$

Assuming that the derivative exists let us denote

$$
\langle A\rangle_{\lambda}=\frac{d}{d \lambda}\left(\operatorname{Tr} E_{\lambda} A\right) .
$$


It follows from the spectral decomposition that the functional $\langle A\rangle_{\lambda}$ on the space of trace-class operators posesses an eigenstate property:

$$
\langle H A\rangle_{\lambda}=\langle A H\rangle_{\lambda}=\lambda\langle A\rangle_{\lambda}
$$

and the spectral theorem may be rewritten in the form

$$
\int_{-\infty}^{\infty}\langle A\rangle_{\lambda} d \lambda=\operatorname{Tr} A
$$

Relations (0.2), (0.3) serve as a motivation for a spectral theorem for deformation quantization.

Let $(M, \omega)$ be a symplectic manifold of dimension $2 n$. It admits the socalled star-product $*$, that is an associative product on formal functions

$$
a(x, h)=\sum_{k=0}^{\infty} h^{k} a_{k}(x)
$$

the series is treated as a formal power series in $h, a_{k}(x) \in C^{\infty}(M)$. The starproduct is given by

$$
a(x) * b(x)=\sum_{k=0}^{\infty} h^{k} C_{k}(a, b)
$$

where $C_{k}(a, b)$ are bidifferential operators $C_{0}(a, b)=a(x) b(x) ; C_{1}(a, b)-$ $C_{1}(b, a)=-i h\{a, b\}$. Here $\{\cdot, \cdot\}$ denotes the Poisson bracket.

Of course, Laurent formal series with finite number of negative degrees are also admissible in (0.4). The algebra of formal functions with a star-product will be denoted $\left(A_{h}, *\right)$. More detail about star-products and their classification one can find in $[1,2]$.

Locally any star-product is equivalent to the Weyl product on a standard symplectic space $\left(\mathbb{R}^{2 n}, \omega_{0}\right)$. It is given by

$$
a(x) * b(x)=\left.\exp \left(-\frac{i h}{2} \omega^{i j} \frac{\partial}{\partial x^{i}} \frac{\partial}{\partial y^{j}}\right) a(x) b(y)\right|_{y=x} .
$$

There is a unique (up to normalization) trace functional on $\left(A_{h}, *\right)$ defined on the ideal $\left(A_{h}^{c}, *\right)$ consisting of functions with compact support. For the Weyl algebra it is simply the integral

$$
\operatorname{Tr} a(x)=\frac{1}{(2 \pi h)^{n}} \int_{\mathbb{R}^{n}} a(x) d x .
$$

Note, that for the Weyl product we have

$$
\operatorname{Tr} a * b=\operatorname{Tr} a b .
$$

After these preliminaries we introduce our main definition. We use the name Hamiltonian for a real function $H(x)$ considered as an element of the algebra $\left(A_{h}, *\right)$. 
Definition 0.1 An eigenstate \langle\rangle$_{\lambda}$ with an eigenvalue $\lambda \in \mathbb{R}$ for a Hamiltonian $H(x)$ is a functional on $\left(A_{h}^{c}, *\right)$

$$
\langle a\rangle_{\lambda}=\sum_{k>>-\infty}^{\infty} h^{k} c_{k}(\lambda)
$$

such that

$$
\langle H * a\rangle_{\lambda}=\langle a * H\rangle_{\lambda}=\lambda\langle a\rangle_{\lambda} .
$$

We say that the spectral theorem holds for $H(x)$ if for any $a \in A_{h}^{c}$

$$
\int_{-\infty}^{\infty}\langle a\rangle_{\lambda} d \lambda=\operatorname{Tr} a .
$$

It is clear that the eigenstate may be multiplied by any number, and (0.9) gives a proper normalization.

Let us briefly describe the content of the paper. We begin with the simplest case when $H(x)$ has no critical point on the support of $a$. In this case the Hamiltonian may be reduced locally to the simplest form for which the eigenstate functional may be constructed explicitly so that (0.9) holds (Theorem 1.2). But this reduction is rather difficult in practice, so we give another construction based on the WKB method (Section 2).

In the next sections we study the spectral theorem in the presens of a critical point of the Morse type, that is an isolated critical point with a nondegenerate second differential. There arise non-trivial contributions of critical points which should be added to the left-hand side of (0.9) to get $\operatorname{Tr} a$ and our aim is to compute these contributions as explicit as possible.

We begin with the case when $H(x)$ is a non-degenerate quadratic form (Section 3). In this case the WKB construction gives an explicit formula for eigenstates in terms of delta functions $\delta^{(k)}(H(x)-\lambda)$.

Further, we investigate the limits

$$
\lim \int_{-\infty}^{-\varepsilon}\langle a\rangle_{\lambda} d \lambda, \quad \lim \int_{\varepsilon}^{\infty}\langle a\rangle_{\lambda} d \lambda
$$

for quadratic Hamiltonians. The explicit formula for the eigenstate allows us to calculate them, using the theory of generalized functions $H_{+}^{\lambda}, H_{-}^{\lambda},(H \pm i 0)$ developed in [4].

In Section 4 we extend this result to an arbitrary Morse critical point. Here the Morse lemma allows us to calculate the limits (0.10) reducing them to the case of quadratic form. The result is quite similar to the case of quadratic Hamiltonians except that the contributions of critical points are not so explicit.

We also discuss briefly the perturbation theory for our problem. It gives a reasonable formula for the contribution of the Morse critical point, but the 
procedure is by no means rigorous from mathematical point of view. The question arises if the two formulas are the same.

Finally, in the last section we discuss very briefly the relation to spectral asymptotics [5]. This is done on an example of harmonic oscillator where everything may be calculated explicitly. This example illustrates also relations of egenstates to the index theory in deformation quantization.

I would like to express my profound gratitude to N. Tarkhanov for his interest and helpful assistance.

\section{Non-critical case}

We deal mostly with a local picture, so the algebra $\left(A_{h}, *\right)$ will be the Weyl algebra with the product $(0.5)$. Let $H(x)$ be a real function and $x_{0}$ be its non-critical point, that is the differential $d H\left(x_{0}\right)$ does not vanish.

Lemma 1.1 In a neighborhood of a non-critical point there exists an isomorphism of the algebra $\left(A_{h}, *\right)$ which sends the function $H(x)$ to a linear function $\left(\operatorname{say} x^{1}\right)$.

Proof. It is well known that there exist Darboux local coordinates for which $H(x)=x^{1}$. The corresponding isomorphism of $\left(A_{h}, *\right)$ (see e.g. [2]) gives a function which differs from $x^{1}$ by higher-order terms, so that

$$
H(x, h)=x^{1}+h H_{1}(x)+h^{2} H_{2}(x)+\ldots
$$

But the higher-order terms may be killed by a conjugation isomorphism

$$
U^{-1} * x^{1} * U=x^{1}+h H_{1}+h^{2} H_{2}(x)+\ldots
$$

For the Weyl *-product we get

or

$$
-i h U^{-1} * \omega^{1 i} \frac{\partial U}{\partial x^{i}}=h H_{1}+h^{2} H_{2}+\ldots
$$

$$
-i \omega^{1 i} \frac{\partial U}{\partial x^{i}}=U *\left(H_{1}+h H_{2}+\ldots\right)
$$

This equation may be easily solved by integration along trajectories of the vector field $\omega^{1 i} \partial / \partial x^{i}$.

$$
\begin{aligned}
& \text { Theorem 1.2 For } H(x)=x^{1} \in\left(A_{h}, *\right) \text { the eigenstate functional is given } \\
& \qquad \begin{aligned}
\langle a(x)\rangle_{\lambda}=(2 \pi h)^{-n}\left(\delta\left(x^{1}-\lambda\right), a(x)\right) \\
=(2 \pi h)^{-n} \int_{\mathbb{R}^{2 n-1}} a\left(\lambda, x^{2}, \ldots, x^{2 n}\right) d x^{2} \ldots d x^{2 n} .
\end{aligned}
\end{aligned}
$$

This is a unique functional with the properties (0.8), (0.9). 
Proof. The eigenstate property follows from

$$
\begin{aligned}
& \left(x^{1}-\lambda\right) * a(x)=\left(x^{1}-\lambda\right) a(x)-\frac{i h}{2} \omega^{1 i} \frac{\partial a}{\partial x^{i}} \\
& a(x) *\left(x^{1}-\lambda\right)=\left(x^{1}-\lambda\right) a(x)+\frac{i h}{2} \omega^{1 j} \frac{\partial a}{\partial x^{j}} .
\end{aligned}
$$

The leading term of $\langle(H-\lambda) * a\rangle_{\lambda}$ is zero because $\left(x^{1}-\lambda\right) \delta\left(x^{1}-\lambda\right)=0$, and the second term vanishes under integration over $x^{i}(i \neq 1)$.

Further,

$$
\frac{1}{(2 \pi h)^{n}} \int_{-\infty}^{\infty}\left(\delta\left(x^{1}-\lambda\right), a(x)\right) d \lambda=\frac{1}{(2 \pi h)^{n}} \int_{\mathbb{R}^{2 n}} a\left(\lambda, x^{2}, \ldots, x^{2 n}\right) d \lambda d x^{2} \ldots d x^{2 n}
$$

which is actually the trace of $a$.

To prove uniqueness, observe that the eigenstate properties (0.8), (0.9) imply

$$
\operatorname{Tr} p\left(x^{1}\right) * a(x)=\int_{\mathbb{R}} p(\lambda)\langle a\rangle_{\lambda} d \lambda,
$$

for any polynomial $p(\lambda)$. Thus $\langle a\rangle_{\lambda}$ is unique since the trace is unique and polynomials are dense.

The construction of $\langle a\rangle_{\lambda}$ does not depend on the choices of local Darboux coordinates and isomorphisms. This may be proved in a standard way similarly to the trace construction (see [2]). Now, using partition of unity in the algebra $\left(A_{h}, *\right)$, one can construct an eigenstate for a function with an arbitrary compact support not containing critical points of $H$. We will not consider such a global construction here, though it is, of course, very important and interesting.

\section{WKB eigenstate construction}

Theorem 1.2 gives a construction of an eigenstate, but it is quite impossible to use it in practice. Now we give a more convenient formula. It is based on the Schrödinger evolution equation

$$
\frac{d U}{d t}-\frac{i}{h} H * U=0,\left.\quad U\right|_{t=0}=1 .
$$

There exist certain difficulties for giving a rigorous meaning to the solution $U$. A special name for it (star-exponential) and the notation $U=\exp _{*}\left(\frac{i}{h} H t\right)$ does not clarify its meaning since the series for the exponential function is 
meaningless even as a formal series. We will consider $U$ as a formal solution of a special form

$$
U=e^{\frac{i}{h} S(x, t)} V(x, t, h)=e^{\frac{i}{h} S}\left(V_{0}+h V_{1}+h^{2} V_{2}+\ldots\right) .
$$

The word "formal" means that if we take the $N$-th truncation

$$
\left.U\right|_{N}:=e^{\frac{i}{h} S}\left(V_{0}+h V_{1}+\ldots+h^{N} V_{N}\right)
$$

and substitute it into (2.1) the result will be of the form

$$
h^{N+1} e^{\frac{i}{h} S}\left(W_{0}+h W_{1}+\ldots\right)
$$

with a formal power series $W$ in positive powers of $h$.

But first we need to extend the Weyl star-product $*$ to the functions containing factors $e^{i S / h}$, taking care of the associativity. This may be done with the help of the following trick which is often used in deformation quantization. First we treat $h$ as a positive number and smooth functions $a(x), b(x)$ as Weyl symbols of pseudo-differential operators. Then there is an integral composition formula for symbols [2]

$$
(a * b)(z, h)=(\pi h)^{-2 n} \int_{\mathbb{R}^{4 n}} \exp \left(\frac{2 i}{h} \omega(u, v)\right) a(z+u) b(z+v) d u d v .
$$

Its stationary phase expansion at $h \rightarrow+0$ gives an asymptotic series in powers of $h$ which, when treated as a formal one, gives the star-product (0.5). The integral formula (2.3) remains meaningful for the functions of the type $e^{i S(x) / h} V(x)$, since for fixed $h \in(0,1]$ such an exponent is a usual smooth function. But now stationary phase expansion of the integral

$$
\begin{aligned}
& H(z) *\left(e^{\frac{i}{h} S(z)} V(z)\right) \\
& =(\pi h)^{-2 n} \int_{\mathbb{R}^{4 n}} \exp \left(\frac{i}{h}(2 \omega(u, v)+S(z+v))\right) H(z+u) V(z+v) d u d v
\end{aligned}
$$

takes the form slightly different from (0.5).

Let us calculate a few first terms. The stationary point of the phase function

$$
2 \omega(u, v)+S(z+v)
$$

is $u=1 / 2 \nabla S, v=0$ where $\nabla S$ is the symplectic gradient

$$
\nabla^{i} S=\omega^{i j} \frac{\partial S}{\partial z^{j}}
$$

Now,

$$
S(z+v)=S(z)+\frac{\partial S(z)}{\partial z^{i}} v^{j}+\frac{1}{2} \frac{\partial^{2} S(z)}{\partial z^{i} \partial z^{j}} v^{i} v^{j}+R_{3}(z, v)
$$


where the remainder $R_{3}$ has a third-order zero at $v=0$. Thus, the phase function may be rewritten in the form

$$
S(z)+2 \omega\left(\left(u-\frac{1}{2} \nabla S(z)-\frac{1}{4} \frac{\partial \nabla S(z)}{\partial z^{k}} v^{k}\right), v\right)+R_{3}(z, v) .
$$

Taking the expression in parentheses as a new variable $\tilde{u}$, we reduce the integral (2.4) to the form

$$
\begin{gathered}
e^{\frac{i}{h} S(z)}(\pi h)^{-2 n} \int_{\mathbb{R}^{4 n}} \exp \left(\frac{2 i}{h} \omega(\tilde{u}, v)\right) e^{\frac{i}{h} R_{3}(z, v)} \\
H\left(z+\frac{1}{2} \nabla S+\tilde{u}+\frac{1}{4} \frac{\partial \nabla S}{\partial z^{k}} v^{k}\right) V(z+v) d \tilde{u} d v .
\end{gathered}
$$

To obtain the stationary phase expansion, we need to expand the integrand into the formal Taylor series in $\tilde{u}, v$ (except the factor $\exp (2 i / h \omega(\tilde{u}, v))$ and integrate termwise. The result may be written as follows

$$
\left.e^{\frac{i}{h} S(z)} \sum_{|\alpha|=0}^{\infty} \frac{1}{\alpha !} H^{(\alpha)}(y+u) *\left(\left(\frac{1}{4} \frac{\partial \nabla S(z)}{\partial z^{k}} u^{k}\right)^{\alpha} e^{\frac{i}{h} R_{3}(z, u)} V(z+u)\right)\right|_{u=0}
$$

where $*$ means the star-product $(0.5)$ with respect to the variable $u \in \mathbb{R}^{2 n}$ and we use the notation

$$
y=z+\frac{1}{2} \nabla S(z)
$$

Now we return to the formal series and treat (2.5) as a formal expansion of (2.3) obtaining the desired extension of the Weyl star-product. We will call this trick a formal stationary phase expansion. The extended product $*$ is associative since it comes from the associative operator product. Moreover, the property (0.6) is still fulfilled since it holds for the integral formula (2.3). Indeed, denoting inessential normalizing factors by $c$, we obtain

$$
\begin{aligned}
& \operatorname{Tr} a * b=c \int_{\mathbb{R}^{6 n}} \exp \left(\frac{2 i}{h} \omega(u, v)\right) a(z+u) b(z+v) d u d v d z \\
& =c \int_{\mathbb{R}^{6 n}} \exp \left(\frac{2 i}{h} \omega(u, w-z)\right) a(z+u) b(w) d u d z d w .
\end{aligned}
$$

Integration in $u$ and $z$ gives $a(w)$, so the result will be the integral over $w$ of $a(w) b(w)$.

Now we construct a formal solution of (2.1) using a well-known WKB method adopted to the Weyl calculus.

Theorem 2.1 There exists a unique formal solution of the Schrödinger equation defined on a small neighborhood of $\left(x_{0}, 0\right) \in \mathbb{R}^{2 n+1}$. 
Proof. Consider the Hamiltonian flow $y=f(x, t)$ defined by the Hamiltonian function $H(x)$ in a neighborhood of a point $x_{0} \in \mathbb{R}^{2 n}$. It is a symplectomorphism, provided $t$ is sufficiently small $(|t|<\varepsilon)$. For a trajectory $y(\tau), \tau \in[0, t]$ we denote by $x=y(0)$ its initial point, $y=y(t)=f(x, t)$ its end point and by $z=z(t)=(x+y) / 2$ its middle point (not necessarily belonging to the trajectory). The trajectory $0 \leq \tau \leq t$ is uniquely defined by its middle point $z$ and $t \in[0, \varepsilon]$. Introduce the function

$$
S(z, t)=\int_{0}^{t}\left(\frac{1}{2} \omega(\dot{y}(\tau), y(\tau)-x)+H(y(\tau))\right) d \tau,
$$

that is, the action along the trajectory with the middle point $z$. It is a generating function of the symplectomorphism $y=f(x, t)$ (see e. g. [3]), that is

$$
\nabla S(z, t)=y-x
$$

or

$$
x=z-\frac{1}{2} \nabla S(z, t), \quad y=z+\frac{1}{2} \nabla S(z, t) .
$$

Finally, it satisfies the Hamilton - Jacobi equation in the form

$$
\frac{\partial S(z, t)}{\partial t}=H\left(z+\frac{1}{2} \nabla S(z, t)\right) \equiv H(y), \quad S(z, 0)=0
$$

(note, that the function $S(z, t)$ introduced here differs from that in [3] by the factor -2 ).

Let us look for a solution in the form (2.2). Substituting it into the Schrödinger equation (2.1) and using composition formula (2.5), we obtain

$$
\begin{aligned}
& \left(\frac{i}{h} \frac{\partial S}{\partial t} V+\frac{\partial V}{\partial t}\right) \\
& =\left.\frac{i}{h} \sum_{|\alpha|=0}^{\infty} H^{(\alpha)}(y+u) *\left(\left(\frac{1}{4} \frac{\partial \nabla S}{\partial z^{k}} u^{k}\right)^{\alpha} e^{\frac{i}{h} R_{3}(z, u)} V(z+u)\right)\right|_{u=0} .
\end{aligned}
$$

Now, for $V=V_{0}+V_{1}+\ldots$ the leading term in (2.7) gives the Hamilton - Jacobi equation (2.6), so that $S(z, t)$ is the generating function of the Hamiltonian flow. The next term of degree 0 in $h$ gives an equation for $V_{0}$ :

$$
\begin{aligned}
& \frac{\partial V_{0}(z, t)}{\partial t}+\frac{1}{2} \nabla^{i} H(y) \frac{\partial V_{0}(z, t)}{\partial z^{i}} \\
& =\frac{1}{8} \omega^{i k} \omega^{j l} \frac{\partial^{2} H}{\partial y^{i} \partial y^{j}}(y) \frac{\partial^{2} S(z, t)}{\partial z^{k} \partial z^{l}} V_{0},
\end{aligned}
$$

which may be rewritten as

$$
\frac{d}{d t} V_{0}(z(t), t)=a(t) V_{0}(z(t), t),\left.\quad V_{0}\right|_{t=0}=1
$$


where

$$
a(t)=\frac{1}{8} \omega^{i j} \omega^{k l} \frac{\partial^{2} H}{\partial y^{i} \partial y^{k}}(y(t)) \frac{\partial^{2} S}{\partial z^{j} \partial z^{l}}(z(t), t)
$$

is a known function since $S(z, t)$ was found previously.

Further equations for $V_{1}, V_{2}, \ldots$ are similar to (2.8), namely,

$$
\frac{d}{d t} V_{k}(z(t), t)=a(t) V_{k}(z(t), t)+b_{k}(t),\left.\quad V_{k}\right|_{t=0}=0
$$

with the same coefficient $a(t)$ and known functions $b_{k}(t)$ which may be expressed from (2.7) in terms of $H, S, V_{0}, V_{1}, \ldots, V_{k-1}$ found previously.

To construct an eigenstate functional $\langle a\rangle_{\lambda}$ on functions $a(x)$ supported in a small neighborhood of a non-critical point $x_{0}$ of the Hamiltonian $H(x)$, we take a cut-off function $f(t)$ vanishing outside the interval $|t|<\varepsilon$ and equal identically to 1 in a smaller neighborhood of $t=0$.

Theorem 2.2 The eigenstate functional is given by a formal stationary phase expansion of the integral

$$
\langle a\rangle_{\lambda}=\frac{1}{(2 \pi h)^{n+1}} \int_{\mathbb{R}^{2 n+1}} f(t) \exp \left(\frac{i}{h}(S(z, t)-\lambda t)\right) V(z, t, h) a(z) d z d t .
$$

It has the form

$$
\begin{aligned}
& \langle a\rangle_{\lambda}=\frac{1}{(2 \pi h)^{n}} \sum_{k=0}^{\infty}(i h)^{k} \frac{\partial^{k}}{\partial \lambda^{k}}\left(\delta(H(z)-\lambda), a_{k}(z)\right) \\
& =\frac{1}{(2 \pi h)^{n}} \sum_{k=0}^{\infty}(-i h)^{k}\left(\delta^{(k)}(H(z)-\lambda), a_{k}(z)\right)
\end{aligned}
$$

where

$$
a_{k}(z)=\left.\frac{1}{k !} \frac{\partial^{k}}{\partial t^{k}} e^{\frac{i}{h} R_{3}(z, t)} V(z, t, h) a(z)\right|_{t=0} .
$$

Note, that only positive degrees of $h$ occur in (2.11) since $R_{3}$ has a thirdorder zero at $\mathrm{t}=0$.

Proof. Let us calculate the formal stationary phase expansion. Stationary points of the phase function $S(z, t)-\lambda t$ should satisfy the equation

$$
\nabla S(z, y) \equiv f(x, t)-x=0
$$

which is possible only for $t=0$. The derivative in $t$ also should vanish, so in virtue of $(2.6)$

$$
\frac{\partial S}{\partial t}=H(y)-\lambda=0
$$


The second derivative $\frac{\partial^{2} S}{\partial t^{2}}$ also vanishes at $t=0$. Indeed,

$$
\frac{\partial^{2} S}{\partial t^{2}}=\frac{\partial}{\partial t} H\left(z+\frac{1}{2} \nabla S(z, t)\right)=\frac{1}{2} \frac{\partial H(y)}{\partial y^{i}} \nabla^{i} \frac{\partial S}{\partial t} .
$$

Thus at $t=0$ we have

$$
\left.\frac{\partial^{2} S}{\partial t^{2}}\right|_{t=0}=\frac{1}{2} \frac{\partial H(z)}{\partial z^{i}} \omega^{i j} \frac{\partial H(z)}{\partial z^{j}}=0
$$

so, that the Taylor formula at $t=0$ gives

$$
S(z, t)-\lambda t=(H(z)-\lambda) t+R_{3}(z, t)
$$

where $R_{3}(z, t)$ has a third-order zero at $t=0$.

Using (2.13), the integral (2.10) may be rewritten in the form

$$
\begin{aligned}
& \langle a\rangle_{\lambda}=\frac{1}{(2 \pi h)^{n+1}} \int_{-\infty}^{\infty} d t \int_{-\infty}^{\infty} d H f(t) e^{\frac{i}{h}(H-\lambda) t} \\
& \left(\delta(H(z)-H), e^{\frac{i}{h} R_{3}(z, t)} V(z, t, h) a(z)\right)
\end{aligned}
$$

where $\delta(H(z)-H)$ is the $\delta$-function on a smooth surface $H(z)=H$, as defined in [4]. To get the stationary phase expansion, we take the formal Taylor series of the function

$$
e^{\frac{i}{h} R_{3}(z, t)} V(z, t, h) a(z)=\sum_{k=0}^{\infty} a_{k}(z) t^{k}
$$

and integrate it termwise. Using that

$$
t^{k} e^{\frac{i}{h}(H-\lambda) t}=\left(i h \frac{\partial}{\partial \lambda}\right)^{k} e^{\frac{i}{h}(H-\lambda) t}
$$

and

$$
\int_{-\infty}^{\infty} f(t) e^{\frac{i}{h}(H-\lambda) t} d t=2 \pi h \delta(H-\lambda)+O\left(h^{\infty}\right)
$$

we come to $(2.11)$.

It remains to show that $\langle a\rangle_{\lambda}$ actually is an eigenstate. To prove $(0.9)$, observe that the terms with $k \neq 0$ vanish under integration over $\lambda$ since they are derivatives in $\lambda$ of functions with compact support. So,

$$
\begin{aligned}
& \int_{-\infty}^{\infty}\langle a\rangle_{\lambda}=\frac{1}{(2 \pi h)^{n}} \int_{-\infty}^{\infty}(\delta(H(z)-\lambda), a(z)) d \lambda \\
& =\frac{1}{(2 \pi h)^{n}} \int_{\mathbb{R}^{2 n}} a(z) d z=\operatorname{Tr} a .
\end{aligned}
$$


To prove (0.8), rewrite $(2.10)$ in the form

$$
\frac{1}{2 \pi h} \int_{-\infty}^{\infty} d t f(t) \operatorname{Tr}\left(U(z, t, h) e^{-\frac{i}{h} \lambda t} a(z)\right) .
$$

Now, substituting $(H-\lambda) * a$ instead of $a$ into (2.14), we would have

$$
\begin{aligned}
& \operatorname{Tr}\left(U e^{-\frac{i}{h} \lambda t}((H-\lambda) * a)\right)=\operatorname{Tr}\left(U e^{-\frac{i}{h} \lambda t} *(H-\lambda) * a\right) \\
& =-i h \frac{d}{d t} \operatorname{Tr}\left(U e^{-\frac{i}{h} \lambda t} * a\right)=-i h \frac{d}{d t} \operatorname{Tr}\left(U e^{-\frac{i}{h} \lambda t} a\right) .
\end{aligned}
$$

Here we have used the property (0.6) and the Schrödinger equation (2.1). So, after integration by parts in $t$ the integral (2.14) becomes

$$
\frac{i}{2 \pi} \int_{-\infty}^{\infty} f^{\prime}(t) \operatorname{Tr}\left(U e^{-\frac{i}{h} \lambda t} a\right) d t .
$$

This integral is $O\left(h^{\infty}\right)$ since there are no stationary points of the phase function on the support of $f^{\prime}(t) a(z)$. So, treated as a formal power series in $h$, it is equal to 0 .

\section{Quadratic Hamiltonians}

Let $H(x)$ be a non-degenerate quadratic form which we will write as

$$
H(x)=\frac{1}{2} g_{i j} x^{i} x^{j}=\frac{1}{2} \omega_{i k} a_{j}^{k} x^{i} x^{j}=\frac{1}{2} \omega(x, A x)
$$

where $A$ is an infinitesimally symplectic matrix. The corresponding Hamiltonian flow is a linear transformation of $\mathbb{R}^{2 n}$ given by the matrix $e^{A t}$.

The WKB method for quadratic Hamiltonians gives a precise solution of the Schrödinger equation. The action has the form

$$
S(z, t)=\omega(z, \tanh (A t / 2) z)
$$

and the amplitude $V_{0}$ depends only on $t$

$$
V_{0}(t)=\operatorname{det}^{-1 / 2}(\cosh (\mathrm{At} / 2)) .
$$

As to $V_{1}, V_{2}, \ldots$, they all vanish since the functions $b_{1}, b_{2}, \ldots$ in $(2.9)$ turn out to be identically 0 .

The expressions (2.11), (2.12) for the eigenstate take more convenient form due to the special structure of the action. Indeed, introducing a matrix-valued function

$$
d(t)=\left(\frac{\tanh (A t / 2)}{A t / 2}\right)^{-1 / 2}
$$


rewrite (3.1) in the form

$$
S(z, t)=\frac{1}{2} \omega\left(d^{-1}(t) z, A\left(d^{-1}(t) z\right)\right) t=H\left(d^{-1}(t) z\right) t .
$$

Thus, changing variables $z=d(t) u$, we obtain

$$
\operatorname{Tr}\left(U e^{-\frac{i}{h} \lambda t} a\right)=\frac{1}{(2 \pi h)^{n}} \int_{\mathbb{R}^{2 n}} e^{\frac{i}{h}(H(u)-\lambda) t} V_{0}(t) \operatorname{det} d(t) a(d(t) u) d u .
$$

Now, similarly to Theorem 2.2 , we come to a modified formula for eigenstates.

Theorem 3.1 The functional $\langle a\rangle_{\lambda}$ for $\lambda \neq 0$ is given by the formula (2.11) where

$$
a_{k}(z)=\left.\frac{1}{k !} \frac{\partial^{k}}{\partial t^{k}}\left(V_{0}(t) \operatorname{det}^{-1 / 2} d(t)\right) a(d(t) z)\right|_{t=0}
$$

Remark 3.2 The functions

$$
\operatorname{det} d(t)=\operatorname{det}^{-1 / 2}\left(\frac{\tanh A t / 2}{A t / 2}\right)
$$

and

$$
k(t)=V_{0}(t) \operatorname{det} d(t)=\operatorname{det}^{-1 / 2}\left(\frac{\sinh A t / 2}{A t / 2}\right)
$$

are known in the theory of characteristic classes. The first one generates the so-called Hirzebruch L-genus and the second the Atiyah-Hirzsbruch $\widehat{A}$-class. To our mind, this curious fact should be investigated deeper.

Our next goal is to calculate the contribution $B(0)$ of the critical point $z=0$ to the left-hand side of $(0.9)$.

Theorem 3.3 The spectral theorem for quadratic Hamiltonians has the form

$$
\operatorname{Tr} a=\text { v.p. } \int_{-\infty}^{\infty}\langle a\rangle_{\lambda} d \lambda+(B(0), a)
$$

where

$$
\begin{aligned}
& (B(0), a)= \\
& \cos \frac{\pi q}{2} \sum_{k=n}^{\infty} \frac{h^{k-n}}{(k-n) ! \sqrt{|\operatorname{det} g|}}\left(\left(\frac{1}{2} g^{i j} \frac{\partial^{2}}{\partial x^{i} \partial x^{j}}\right)^{k-n} \delta(x), a_{k}(x, h)\right)
\end{aligned}
$$

Here $q$ is the number of negative squares in the canonical form of $H(x)$ (inertia index) and $a_{k}$ are given by (3.3). In particular, for $q$ odd the contribution of the critical point at the origin is equal to 0. 
Proof. We have

$$
(B(0), a)=\lim _{\varepsilon \rightarrow+0}\left(\operatorname{Tr} a-\int_{\varepsilon}^{\infty}\langle a\rangle_{\lambda} d \lambda-\int_{-\infty}^{-\varepsilon}\langle a\rangle_{\lambda} d \lambda\right)
$$

where all the operations over formal series such as integration or passing to the limit are understood as termwise. The leading term up to a factor $(2 \pi h)^{-n}$ gives

$$
\lim _{\varepsilon \rightarrow+0} \int_{\varepsilon}^{\infty}(\delta(H(x)-\lambda), a(x)) d \lambda=\int_{H(x)>0} a(x) d x
$$

and similarly

$$
\lim _{\varepsilon \rightarrow+0} \int_{-\infty}^{-\varepsilon}(\delta(H(x)-\lambda), a(x)) d \lambda=\int_{H(x)<0} a(x) d x .
$$

Their sum (multiplied by $(2 \pi h)^{-n}$ ) coincides with the trace, so (3.6) may be rewritten in the form

$$
\begin{aligned}
& (B(0), a)=\left.(2 \pi h)^{-n} \sum_{k=1}^{\infty}(i h)^{k} \lim _{\varepsilon \rightarrow+0} \frac{\partial^{k-1}}{\partial \lambda^{k-1}}\left(\delta(H(x)-\lambda), a_{k}(x)\right)\right|_{\lambda=-\varepsilon} ^{\lambda=\varepsilon} \\
& =(2 \pi h)^{-n} \sum_{k=1}^{\infty}(i h)^{k} \lim _{\varepsilon \rightarrow+0}\left(\frac{\partial^{k-1}}{\partial \varepsilon^{k-1}}\left(\delta(H(x)-\varepsilon), a_{k}(x)\right)\right. \\
& \left.+(-1)^{k} \frac{\partial^{k-1}}{\partial \varepsilon^{k-1}}\left(\delta(-H(x)-\varepsilon), a_{k}(x)\right)\right) .
\end{aligned}
$$

Here we have used the identity

$$
\delta(H(x)+\varepsilon)=\delta(-H(x)-\varepsilon) .
$$

To calculate the limit, we pass over to the Mellin transform

$$
\begin{aligned}
& M_{k}^{ \pm}(\zeta)=\int_{0}^{\infty} \varepsilon^{\zeta-1} \frac{\partial^{k-1}}{\partial \varepsilon^{k-1}}\left(\delta( \pm H(x)-\varepsilon), a_{k}(x)\right) d \varepsilon \\
& =(-1)^{k-1}(\zeta-1)(\zeta-2) \ldots(\zeta-k+1) \int_{0}^{\infty} \varepsilon^{\zeta-k}\left(\delta( \pm H(x)-\varepsilon), a_{k}(x)\right) d \varepsilon \\
& =(-1)^{k-1}(\zeta-1)(\zeta-2) \ldots(\zeta-k+1) \int_{ \pm H(x)>0}( \pm H(x))^{\zeta-k} a_{k}(x) d x \\
& =(-1)^{k-1}(\zeta-1)(\zeta-2) \ldots(\zeta-k+1)\left(H_{ \pm}^{\zeta-k}(x), a_{k}(x)\right) .
\end{aligned}
$$

These functions are meromorphic with the poles at most of the second order [4]. If inertia index $q$ is odd, then $M_{k}^{ \pm}$have the second-order pole at $\zeta=0$, so the limits $(0.10)$ do not exist. For $q$ even $\zeta=0$ is a simple pole, thus, the limits exist and are equal to the residues of $M_{k}^{ \pm}$at $\zeta=0$. 
Fortunately, the functions

$$
M_{k}^{+}(\zeta)+(-1)^{k} M_{k}^{-}(\zeta)
$$

needed for the contribution (3.7) behave better. Namely, the functions

$$
(H \pm i 0)^{\zeta}=H_{+}^{\zeta}+e^{ \pm i \pi \zeta} H_{-}^{\zeta}
$$

turn out to have only simple poles at $\zeta=-n,-n-1,-n-2, \ldots$ Thus,

$$
H_{+}^{\zeta-k}+(-1)^{k} H_{-}^{\zeta-k}=\frac{e^{-\frac{i \pi \zeta}{2}}(H+i 0)^{\zeta-k}+e^{\frac{i \pi \zeta}{2}}(H-i 0)^{\zeta-k}}{2 \cos \frac{\pi \zeta}{2}}
$$

has a simple pole at $\zeta=0$ for $k=n, n+1, \ldots$ with the residue [4]

$$
\begin{aligned}
& \operatorname{res}\left(H_{+}^{\zeta-k}+(-1)^{k} H_{-}^{\zeta-k}\right)=\frac{1}{2} \operatorname{res}\left((H+i 0)^{\zeta-k}+(H-i 0)^{\zeta-k}\right) \\
& =\frac{(2 \pi)^{n} \cos \frac{\pi q}{2}}{(k-n) !(k-1) ! \sqrt{|\operatorname{det} g|}}\left(\frac{1}{2} g^{i j} \frac{\partial^{2}}{\partial x^{i} \partial x^{j}}\right)^{k-n} \delta(x) .
\end{aligned}
$$

This completes the proof.

\section{Morse critical points}

We are ready now to discuss a general case of the Morse critical point. This is an isolated critical point with a non-degenerate second differential (Hessian)

$$
\left.d^{2} H\right|_{x_{0}}=\frac{1}{2} g_{i j} d x^{i} d x^{j}
$$

The Morse lemma reads that there are local coordinates for which the Hamiltonian $H(x)$ becomes precisely the quadratic form (4.1):

$$
H(f(y))=\frac{1}{2} g_{i j} y^{i} y^{j}
$$

where $f$ is a local diffeomorphism. Unfortunately, $f$ is not a symplectomorphism in general, so we cannot automatically reduce the general case to quadratic Hamiltonians considered in Section 3. Nevertheless, Theorem 3.3 admits a generalization to the case of an arbitrary Morse critical point. 
Theorem 4.1 The spectral theorem in the presence of the Morse critical point has the form

$$
\begin{aligned}
& \operatorname{Tr} a=\text { v.p. } \int_{-\infty}^{\infty}\langle a\rangle_{\lambda} d \lambda+\cos \frac{\pi q}{2} \sum_{k=n}^{\infty} \frac{h^{k-n}}{(k-n) ! \sqrt{|\operatorname{det} g|}} \\
& \left(\left(\frac{1}{2} g^{i j} \frac{\partial^{2}}{\partial y^{i} \partial y^{j}}\right)^{k-n} \delta(y), a_{k}(f(y)) \operatorname{det} \frac{\partial f}{\partial y}\right)
\end{aligned}
$$

where $a_{k}(z)$ are given by (2.12) and $f$ is the diffeomorphism (4.2).

Proof. We start with the relations (2.11), (2.12) valid for any Hamiltonian $H(x)$ and a non-critical value $\lambda$ and proceed similarly to the proof of Theorem 3.3. The relation (3.7) holds true no matter whether $H(x)$ is a quadratic form or not. Thus, the limit of the $k$-th summand in (3.7) is equal to

$$
\begin{aligned}
& (i h)^{k} \operatorname{res}\left(M_{k}^{+}(\zeta)+(-1)^{k} M_{k}^{-}(\zeta)\right) \\
& =(i h)^{k}(k-1) ! \operatorname{res}\left(\left(H_{+}^{\zeta-k}, a_{k}\right)+(-1)^{k}\left(H_{-}^{\zeta-k}, a_{k}\right)\right)
\end{aligned}
$$

precisely as in the case of quadratic Hamiltonians. Now, the generalized function $H_{ \pm}^{\zeta}$ is defined by the integral

$$
\left(H_{ \pm}^{\zeta}, \varphi\right)=\int_{ \pm H(x)>0}( \pm H)^{\zeta}(x) \varphi(x) d x
$$

and its analytic extension. At this step the reduction to the quadratic form is possible: we simply change variables in the integral, obtaining

$$
\left(H_{ \pm}^{\zeta}, \varphi\right)=\int_{ \pm H(f(y))>0}( \pm H)^{\zeta}(f(y)) \varphi(f(y)) \operatorname{det} \frac{\partial f}{\partial y} d y .
$$

The rest of the proof goes as in Theorem 3.3, but the test function $\varphi(x)$ should be replaced by $\varphi(f(y)) \operatorname{det} \frac{\partial f}{\partial y}$.

In the rest of this section we consider another approach to compute the contribution of the Morse critical point. It is known in physics literature as perturbation theory. Mathematically it is not quite rigorous, but it also gives a reasonable answer which is interesting to compare with the rigorous result. On the other hand, the calculations here seem to be more transparent than in the approach based on WKB-method and Morse lemma.

We represent the Hamiltonian in the form

$$
H=H_{0}(x)+H_{1}(x)
$$

where $H_{0}(x)=\omega(x, A x) / 2$ is a non-degenerate quadratic form and $H_{1}(x)$ is a "perturbation", that is a function with the third-order zero at the origin. 
Now, we try to solve the Schrödinger equation

$$
\frac{d U}{d t}=\left(\frac{i}{h} H_{0}+\frac{i}{h} H_{1}\right) * U, \quad U(0)=1
$$

using the explicit solution

$$
U_{0}=\operatorname{det}^{-1 / 2}(\cosh (A t / 2) x) \exp \left(\frac{i}{h} \omega(x, \tanh (A t / 2) x)\right)
$$

for quadratic Hamiltonian $H_{0}$.

To this end we introduce a new grading assigning degree 2 to $h$ and degree $k$ to a function $\varphi(x)$ which has zero of order $k$ at the origin. Thus the term $h^{k} x^{\alpha} \varphi(x)$ where $\alpha$ is a multiindex and $\varphi(0) \neq 0$ has a new degree $2 k+|\alpha|$. Moreover, we shall admit negative powers $k$, provided the total degree is positive. Thus, the degree of $\frac{i}{h} H_{0}$ in (4.3) is equal to 0 , while that of $\frac{i}{h} H_{1}$ is 1 . It is easy to see that the $*$-multiplication respects the grading:

$$
\operatorname{deg}(a * b) \leq \operatorname{deg} a+\operatorname{deg} b .
$$

We look for the solution of (4.3) in the form

$$
U_{0} * V=U_{0} *\left(1+V_{1}+V_{2}+\ldots\right)
$$

where $V_{k}$ has degree $k$. The equation for $V$ looks like

$$
\frac{d V}{d t}=\frac{i}{h}\left(U_{0}^{-1} * H_{1} * U_{0}\right) * V ;\left.\quad V\right|_{t=0}=1
$$

where

$$
\left.\frac{i}{h} U_{0}^{-1} * H_{1} * U_{0}=\frac{i}{h} H_{1}\left(e^{A t} x, h\right):=F_{1}(x, t, h)\right) .
$$

Here $e^{A t}$ is the Hamiltonian flow defined by the quadratic Hamiltonian $H_{0}(x)$. Note that the degree of $F_{1}$ is 1.

The equation (4.4) may be rewritten as

$$
V=1+\int_{0}^{t} F_{1} * V d t_{1}
$$

and may be solved by iterations, the $k$-th iteration being of degree $k$.

After that we formally get an eigenstate $\langle a\rangle_{\lambda}$ in the form (2.11)

$$
\langle a\rangle_{\lambda}=\frac{1}{(2 \pi h)^{n}} \sum_{k=0}^{\infty}(-i h)^{k}\left(\delta^{(k)}\left(H_{0}(x)-\lambda\right), a_{k}\right)
$$

with $a_{k}$ equal to Taylor coefficients at $t=0$ of the function

$$
a(t)=\left.k(t)(V(x, h, t) * a(x))\right|_{x \rightarrow d(t) x}
$$


where $k(t)$ and $d(t)$ are given by $(3.2),(3.4)$ for the Hamiltonian $H_{0}$. Reasoning as in Section 3, we obtain the contribution of the origin in the form

$$
\cos \frac{\pi q}{2} \sum_{k=n}^{\infty} \frac{h^{k-n}}{(k-n) ! \sqrt{|\operatorname{det} g|}}\left(\left(\frac{1}{2} g^{i j} \frac{\partial^{2}}{\partial x^{i} \partial x^{j}}\right)^{k-n} \delta(x), a_{k}(x, h)\right)
$$

with $a_{k}$ defined above.

Observe, that the final result makes sense as a formal series in $h$ only though intermediate steps make no sense in deformation quantization setting, they become meaningful due to the new grading. So, a question arises whether the two formulas for the contribution of the Morse critical point coincide.

\section{Example: spectral asymptotics}

Having eigenstates at his disposal, one may introduce further important spectral notions purely in deformation quantization framework. Let $H(x)$ be a Hamiltonian with a discrete set $\left\{x_{c}\right\}$ of Morse critical points and let $B\left(x_{c}\right)$ denote the contribution of the critical point $x_{c}$.

Definition 5.1 Let $\Delta$ be an interval of the real $\lambda$-axis whose ends are noncritical points of the Hamiltonian.

$A$ formal spectral projector $E(\Delta)$ is a distribution on $a(x) \in A_{h}^{c}$ with values in $\left.\mathbb{C}\left[h^{-1}, h\right]\right]$

$$
(E(\Delta), a(x))=\text { v.p. } \int_{\Delta}\langle a(x)\rangle_{\lambda} d \lambda+\sum_{H\left(x_{c}\right) \in \Delta}\left(B\left(x_{c}\right), a(x)\right) .
$$

In case when $E(\Delta)$ is a distribution with compact support, the expression

$$
\left.(E(\Delta), 1)=\sum_{k>>-\infty}^{\infty} c_{c} h^{k} \in \mathbb{C}\left[h^{-1}, h\right]\right]
$$

will be called a formal spectral asymptotics and denoted by $\operatorname{Tr} E(\Delta)$ or $N(\Delta)$.

These definitions are motivated by analogy with the operator formulas $(0.1),(0.2),(0.3)$ but in fact they have nothing to do with the spectral theory. However, it is instructive to compare these formal spectral objects with genuine ones defined for $h$-pseudo-differential operators [5].

The simplest example for which all the quantities in question may be calculated explicitly is harmonic oscillator

$$
H(x)=\sum_{k=1}^{n} g_{k} \frac{x_{2 k-1}^{2}+x_{2 k}}{2} .
$$


Using (2.11), (3.3), we get

$$
\begin{aligned}
& \langle 1\rangle_{\lambda}=\left.(2 \pi h)^{-n} \sum_{k=0}^{\infty} \frac{(i h)^{k}}{k !} \frac{\partial^{2}}{\partial \lambda \partial t}(\delta(H(x)-\lambda), k(t))\right|_{t=0} \\
& =(2 \pi h)^{-n} k\left(i h \frac{\partial}{\partial \lambda}\right) \frac{\partial}{\partial \lambda}(\theta(\lambda-H(x)), 1) \\
& =\frac{\partial}{\partial \lambda} \frac{1}{g_{1} g_{2} \ldots g_{n}} \prod_{i=1}^{n}\left(\frac{\frac{g_{i} h}{2} \frac{\partial}{\partial \lambda}}{\sinh \frac{g_{i} h}{2} \frac{\partial}{\partial \lambda}}\right) \frac{1}{n !}\left(\frac{\lambda}{h}\right)^{n} .
\end{aligned}
$$

This formula is valid for $\lambda>0$, for $\lambda<0$ we have clearly $\langle a\rangle=0$. Integrating this expression, we see that up to an additive constant the formal spectral asymptotics is given by

$$
N(\lambda)=\frac{1}{g_{1} g_{2} \ldots g_{n}} \prod_{i=1}^{n} \frac{\frac{g_{i} h}{2} \frac{\partial}{\partial \lambda}}{\sinh \frac{g_{i} h}{2} \frac{\partial}{\partial \lambda}} \frac{1}{n !}\left(\frac{\lambda}{h}\right)^{n}
$$

for $\lambda>0$. The constant is determined by the condition

$$
N(\lambda)=\int_{0}^{\lambda}\langle 1\rangle_{\mu} d \mu+(B(0), 1)
$$

where $\mathrm{B}(0)$ is the contribution of the critical point 0 . It follows from (3.5) that the expression (5.3) precisely satisfies this condition. The leading term of (5.3) is $\operatorname{vol}\{H<\lambda\} /(2 \pi h)^{n}$ which coincides with the leading term of the genuine spectral asymptotics. Note however, that the formal $N(\lambda)$ is a polynomial in $\lambda / h$ while the genuine $N(\lambda)$ is certainly not.

If $g_{1}=g_{2}=\ldots=g_{n}=1$, further simplification is possible. Indeed, (5.2) may be rewritten in the form

$$
\left(\frac{\frac{h}{2} \frac{\partial}{\partial \lambda}}{\sinh \frac{h}{2} \frac{\partial}{\partial \lambda}}\right)^{n} \frac{1}{(n-1) !}\left(\frac{\lambda}{h}\right)^{n-1}=\sum_{k=0}^{n-1} c_{k}\left(\frac{\lambda}{h}\right)^{n-1-k} \frac{1}{(n-1-k) !}
$$

where $c_{k}$ are Taylor coefficients of the function $(t / 2 / \sinh t / 2)^{n}$. Thus, (5.4) may be represented as the Taylor coefficient at $t^{n-1}$ of the function

$$
\left(\frac{t / 2}{\sinh t / 2}\right)^{n} e^{\frac{\lambda}{h} t}=\frac{e^{\left(\frac{\lambda}{h}+\frac{n}{2}\right) t}}{\left(e^{t}-1\right)^{n}} t^{n}
$$

that is

$$
\frac{1}{2 \pi i} \int_{|t|=\varepsilon} \frac{e^{\left(\frac{\lambda}{h}+\frac{n}{2}\right) t}}{\left(e^{t}-1\right)^{n}} d t
$$


Changing variables $\zeta=e^{t}-1$, we obtain

$$
\langle 1\rangle_{\lambda}=\frac{1}{2 \pi i} \int_{|\zeta|=\varepsilon} \frac{(1+\zeta)^{\frac{\lambda}{h}+\frac{n}{2}-1}}{\zeta^{n}} d \zeta=C_{\frac{\lambda}{h}+\frac{n}{2}-1}^{n-1} .
$$

The binomial coefficient (5.5) is known to be the index of $\mathbb{C P}^{n-1}$ with the symplectic form equal to the standard Fubini-Study form times $\lambda / h$. This observation allows us to hope that the quantity $\langle 1\rangle_{\lambda}$ may be expressed in terms of characteristic classes similarly to the index in deformation quantization [2].

\section{References}

[1] F. Bayen, M. Flato, C. Fronsdal, A. Lichnerovicz and D. Sternheimer, Deformation theory and quantization, Ann. Physics, 111 (1978), $61-151$.

[2] B. Fedosov, Deformation Quantization and Index Theory, Akademie Verlag, Berlin, 1996.

[3] B. Fedosov, On normal Darboux coordinates, Amer. Math. Soc. Transl. (2), 206, 2002.

[4] I.M. Gelfand and G.E. Shilov, Generalized Functions, vol. 1, Academic Press, New York, 1964.

[5] V. Ivrii, Microlocal Analysis and Precise Spectral Asymptotics, Springer-Verlag, Berlin Heidelberg, 1998.

(B. Fedosov) Moscow Institute

of Electronic Technology,

103498, Moscow, Russia

E-mail address: fedosov@mail.compnet.ru 